\title{
ПРОЦЕССНО-СИСТЕМНЫЙ ПОДХОД В ИССЛЕДОВАНИИ СОЦИАЛЬНО-ЭКОНОМИЧЕСКИХ СИСТЕМ: ОТ КОНЦЕПЦИЙ К МОДЕЛЯМ
}

\author{
О.Н. Поддубная"
}

На основе синтеза современных идей институционально-эволюционного подхода и экономики сложности выносятся на обсуждение новые подходы, расширяющие и развивающие сущностное и содержательное понимание социально-экономических систем. Предложена концептуальная иерархически-сетевая модель «экономическая среда - система - процесс» (EESP), в контексте которой описан процессно-системный подход для исследования динамических и синтетических свойств сложных социально-экономических систем. В рамках динамического подхода описаны общие принципы и технологии идентификации оператора эволюции при моделировании экономической динамики. Постулированы три основных принципа динамической сложности экономических систем в условиях социальных трансформаций. Предложена математическая модель экономической динамики в виде гибридной быстро-медленной системы с запаздыванием и управлением.

Ключевые слова: экономическая динамика, институционально-эволюционное направление, экономика сложности, иерархически-сетевая модель, оператор эволюции, быстро-медленная система.

JEL-классификация: B52, С53, О10.

DOI: $10.46782 / 1818-4510-2021-3-70-82$

Материал поступил 28.04.2021 2.

Еще в начале XX в. Й. Шумпетер отметил, что «новая комбинация средств производства» есть «источник энергии в пределах экономической системы, возмущающий сам по себе любое равновесие, которое может быть достигнуто», фактически определив понятие социально-экономической трансформации и ее причину. На современном этапе под «новой комбинацией средств производства» мы понимаем новую технологию, которая сама является генератором инноваций, порождающих и требующих технологий следующего поколения, в результате чего на современном историческом этапе наблюдается непрерывный ускоряющийся процесс создания и смены технологий. В основе смены технологического уклада лежит переход к новым доминирующим технологиям производства (Глазьев, 1993). В западной литературе для оп- ределения коренных изменений технологий, приводящих к фундаментальным социально-экономическим трансформациям, принято использовать термин «промышленные революции» ${ }^{1}$ В настоящее время масштабные процессы цифровизации формируют новую алгоритмически-цифровую парадигму экономических отношений и жизни общества в целом (Поддубная, 2020). Представители различных научных школ находятся в активном поиске методологических основ, модельных конструкций, инструментов и технологий моделирования для целей и задач многоаспектного анализа социально-экономических систем в условиях цифровой трансформации.

${ }^{1}$ Термин Индустрия 4.0 вошел в широкий оборот на Давосском экономическом форуме в 2016 г. и после издания его основателем К. Швабом монографии «Четвертая промышленная революция».

Поддубная Олеся Николаевна (poddubnaia.o@bseu.by), кандидат физико-математических наук, доцент, Белорусский государственный экономический университет (г. Минск, Беларусь). https://orcid.org/0000-0001-9594-6226 


\section{Гносеологические базисы моделирования әкономической динамики социально-экономических систем}

В рамках системного анализа у любой системы выделяют три группы свойств: статические, динамические и синтетические. Представители классического и неоклассического направлений (именно эти научные школы принято называть ортодоксальным течением) сделали акцент на теории экономического равновесия при условии рационального поведения хозяйствующих субъектов и свободной конкуренции; тем самым исследовательским ядром ортодоксии стало изучение статических свойств социально-экономических систем. В основу научной парадигмы мэйнстрима легли построение моделей состава и структуры изучаемого явления, элегантно формализованных на логико-математическом языке, а также разработка методов решения в рамках этих моделей статических задач для периодов, на протяжении которых взаимное влияние факторов не успевает измениться.

Наиболее значимыми среди гетеродоксальных концепций экономической теории отмечают институциональное и эволюционное направления. Институционализм как самостоятельное влиятельное течение сформировался после издания ключевых работ Р. Коуза, Д. Норта, О. У ильямсона, Э. Остром. K основным положениям институционального направления относят следующие: приоритет анализа социально-экономической системы в целом, а не поведения ее отдельных агентов; акцент на качественном анализе (в отличие от количественного в ортодоксии) результатов, причин, движущих сил и форм хозяйственных изменений с использованием дескриптивных методов (тогда как в мэйнстриме доминирует статистико-математический инструментарий); выделение в качестве объекта исследования многоуровневой институциональной среды с многообразными механизмами регулирования и управления.

Эволюционное направление ориентирует методологию анализа на проблематику долгосрочной эволюции общественных структур, а не на исследование зафиксированных во времени отдельных ситуаций (статических состояний для классики и неоклассики). По мнению основоположников эволюционной экономики Р. Нельсона и С. Уинтера ${ }^{2}$, предпочтения, ресурсы и технологии претерпевают не до конца предсказуемые изменения, ввиду чего оптимум аллокации должен быть подвижен, и речь следует вести о Әинамическом равновесии. Представители эволюционного направления экономической теории в своей исследовательской программе делают акцент на динамических свойствах социально-экономических систем: функциональность, стимулируемость, изменчивость системы со временем, существование в изменяющейся среде.

Экономика сложности возникла фактически как адаптация теории сложности к экономическим проблемам. Взгляд на экономику через призму теории сложности фокусируется на результатах взаимодействий структур, выдвигая на первый план научных исследований синтетические свойства системы: эмерджентность, ингерентность и целесообразность. Экономика сложности «представляет собой иной способ видения экономики - под иным углом зрения, при котором действия и стратегии постоянно развиваются, время становится важным, структуры постоянно формируются и переформировываются, при котором видны феномены, невидимые с позиций стандартного равновесного анализа, и при котором мезоуровень, располагающийся между микро- и макроуровнями, становится важен» (Артур, 2015. С. 17). В рамках теории сложности при развитии системного подхода вслед за кибернетикой рождается новое междисциплинарное направление научных исследований - синергетика. Основной задачей синергетики является изучение процессов на основе общих принципов самоорганизации систем, относящихся к компетенциям различных наук ${ }^{3}$ (то есть

${ }^{2}$ В 1982 г. Р. Нельсоном и С. Уинтером была опубликована совместная работа «Эволюционная теория экономических изменений».

${ }^{3}$ Следует отметить, что основной вклад в продвижение данного научного направления внесли представители естественных наук: немецкой школы лазерной физики (Г. Хакен), российской математической школы нелинейной динамики (А.А. Самарский, С.П. Курдюмов), бельгийской школы квантовой химии (И. Пригожин). 
безотносительно природы систем). С философских позиций синергетику часто определяют как «глобальный эволюционизм» или «универсальную теорию эволюции», подчеркивая единые базисы для объяснения механизмов возникновения любых новаций ${ }^{4}$. Разработанная почти полвека назад исследовательская программа синергетики особенно актуальна в эпоху масштабной делинеаризации. Здесь стоит упомянуть еще одно бурно развивающееся гетеродоксальное направление экономической науки - эконофизику ${ }^{5}$. Проникновение методов физической науки в изучение экономических явлений объясняется особенностями объектов теоретической экономики. Как бы ни отличались их трактовки в разных экономических школах, такими объектами являются производительные силы или экономические ресурсы, необходимые для производства экономических благ и удовлетворения растущих разноплановых потребностей общества. Экономическое развитие сегодня обеспечивается развитием технологий производства, которые базируются на достижениях естествознания, и прежде всего физики и инженерии, поэтому использование методов, инструментов и моделей физической науки для исследования экономических проблем представляется вполне естественным.

В работах широкого круга ученых рассматривается вопрос о разнице синергетического и кибернетического подходов для исследования динамики сложных систем (Жилин, 2010). Кибернетика изучает устой-

${ }^{4}$ В данном определении прослеживается аналогия с определением кибернетики как «универсальной теории управления», подходящей для описания любых операций регулирования в природе, технике, обществе. Философы отмечают эпистемологические новации синергетики и говорят о становлении и развитии синергетического стиля мышления (Зеленков, 2010).

5 Под эконофизикой понимается применение методов теоретической физики для решения прикладных задач экономики. В середине 80-х годов в Нью-Мексико был создан Институт Санта-Фе, который стал одним из главных центров эконофизики, где это направление развивается в рамках общей теории сложности адаптивных систем. Последователи данного направления (Я. Кертес, Ж.-П. Бушо, Ф. Лонгстафф, Г. Стэнли, В. Яковенко, Й. Чжан и др.) отмечают специфику изучения сложных динамических систем при имитационном моделирования. Здесь модели принципов поведения агентов реализуются в виде алгоритма с множеством итераций, конечный результат которых непредсказуем и тем более недоказуем в строгом научном смысле. чивые, управляемые в соответствии с заданной целью динамически равновесные системы. Кибернетический подход в экономических исследованиях делает упор на моделировании сложных систем, которые под воздействием экзогенных факторов (факторов среды) запускают механизмы саморегуляции. Обратные связи стабилизируют систему при воздействии внешнего управления, что выражается в способности системы поддерживать неизменность своих параметров в изменяющейся внешней среде, а для исследователя - в предсказуемости поведения системы. Гносеологические корни синергетического подхода лежат в моделировании динамики неустойчивых, неравновесных, хаотических систем с акцентом на проблеме их самоорганизации. Самоорганизующаяся система без специального вмешательства извне обретает новую пространственную, временную или функциональную структуру (Хакен, 2005). Возникающие неравновесные состояния запускают в кибернетической системе механизмы саморегулящии для обеспечения системной целостности и устойчивости, тогда как для синергетических систем неравновесные состояния служат основанием включения механизмов самоорганизации для спонтанного структурогенеза.

Цифровая трансформация и родственные понятия сегодня широко применяются в социально-экономических исследованиях с использованием пока достаточно разрозненных методологий анализа проблемы. Несмотря на это, большинство ученых сходятся во мнении относительно свойств экономической среды, систем и процессов, формирующихся в условиях цифровой трансформации. Среди них выделяют масштабируемость, универсальность, чрезвычайно высокий динамизм, наукоемкость и интеллектуализацию. Важнейшим гносеологическим аспектом, объединяющим упомянутые три гетеродоксальные направления для целей изучения феноменов цифровой трансформации, является то, что в этих теориях технология рассматривается как основной драйвер изменений в экономике, тогда как в мэйнстриме такая роль отведена более эффективному использованию факторов через саморегуляцию рынка. 
На основе синтеза ключевых положений экономики сложности и институционально-эволюционного направления можно сформулировать парадигмальный тезис о том, что следствием развития социальноэкономических отношений в условиях цифровой трансформации является также и генерация нового понимания социально-экономических систем как эволющионно развивающихся сложных адаптивных систем непрерьвно взаимодействуюших технологических прочессов, регулируемых и управляемых институииональными нормами. Основная часть (до запятой) предложенного определения абстрагирована от конкретных материальных форм. Поэтому в зависимости от понимания механизма адаптации сложной системы как саморегулирующегося или самоорганизующегося для моделирования динамики сложной системы может применяться кибернетический или синергетический подход. Уточняющая часть (после запятой) определения вносит конкретику как в понимание того, что оно сформулировано для социально-экономических систем, так и того, что автор при построении моделей экономической динамики намерен придерживаться кибернетического подхода ввиду крайней важности принципов целеполагания для всех уровней хозяйственной деятельности как основы ее управляемости.

Как отмечал основатель американской школы маржинализма Дж. Б. Кларк, экономическая действительность по своему существу динамична. Н.Д. Кондратьев в докладе «К вопросу о понятиях экономической статики, динамики и конъюнктуры» $(1924$ г.) уточнил, что не следует противопоставлять статику и динамику явлений, а для целей теоретического исследования можно методологически подходить со статической или динамической точки зрения на них. «Под статической мы понимаем теорию, которая рассматривает экономические явления по существу, вне категории изменения их во времени. Наоборот, под динамической мы понимаем ту теорию, которая изучает экономические явления в процессе их изменения во времени. В соответствии с этим для статической точки зрения на экономичес- кую действительность особенно характерной является концепция равновесия взаимно связанных между собою элементов этой действительности. Наоборот, для динамической точки зрения наиболее характерной будет концепция процесса изменений экономических элементов и их связей» (Кондратьев, 1989. С. 49).

Институциональное и эволюционное направления, а также экономика сложности активно и аргументированно отстаивают свою позицию относительно методов исследования социально-экономических явлений и процессов, делая акцент прежде всего на динамических и синтетических свойствах изучаемых систем. Целью данного исследования является развитие методологии процессно-системного подхода (Поддубная, 2020) для моделирования современной экономической динамики на основе синтеза идей экономики сложности и эволюционно-институционального направлений экономической теории.

\section{Иерархически-сетевая модель ЕЕSР}

В указанной выше работе начато обсуждение основных идей процессно-системного подхода и предложена схема иерархической триады «экономическая среда - экономическая система - экономический процесс» с интегрированной в нее экономической динамикой. Иерархическая триада «среда - система - элемент» не нова и является предметной основой системного анализа. Ниже излагаются особенности и основные идеи процессно-системного подхода, трансформирующие данную модель для исследования социально-экономических систем в переходных условиях.

В основе классической интерпретации экономистами ортодоксального направления триады «среда - система - элемент» лежат статические свойства системы: целостность, открытость, структурированность, внутренняя неоднородность (или различимость частей). Поскольку в исследовательской программе статические свойства системы доминируют над динамическими и синтетическими, классическая экономическая теория изобилует иерархическими структурами и факторными моделями со- 
става и структуры. Ключевой бинарной иерархией ортодоксии является дихотомия «микро-макро».

Использование открытий, которые касаются принципов организации, форм, свойств живых популяций (естественных систем), основ их эволюционного развития в технических устройствах 6 и различных искусственных системах, подвигло экономистов обратить более пристальное внимание на динамические свойства социально-экономических систем. Вслед за представителями эволюционного экономического направления последователи неоклассической школы стали признавать фактор времени если не единственным, то ключевым экономическим фактором. Следует отметить, что эволюционная теория, продвигая свои идеи, на сегодняшний день среди немногих гетеродоксальных направлений достаточно хорошо интегрируется в мэйнстрим, поскольку использует типичную для основного русла подачу материала и не отвергает моделирование, часто применяя метафоры, аналогии и методы естественных наук.

В эволюционной экономике крайне редко можно встретить термин «фактор» (кроме временного фактора), которым изобилует классическая теория. Этому терминологическому феномену есть и математическое объяснение. Для последователей эволюционной экономики аксиоматичен тот факт, что любой экономический фактор не стационарен, т. е. это процесс, который может быть формализован функцией, изменяющейся во времени, как и тот факт, что любое экономическое явление в терминах ресурсного, поточного, структурного или бизнес-ориентированного подхода определяется набором факторов, их число чаще всего неопределенно (или бесконечно). Как известно из математического анализа, если рассматривать некоторую функцию нескольких переменных, являющихся функцией одного независимого аргумента $t$, то исходная функция является композицией функций (сложной функцией) одного аргумента $t$. Какой бы сложной ни была фун-

${ }^{6}$ В 1960 г. в Дайтоне (США) состоялся первый симпозиум по бионике - междисциплинарной науке, решающей инженерные задачи на основе обнаруженных в живой природе принципов. кциональная зависимость факторов, описывающих любое экономическое явление, она с учетом рекурсивного применения описанной выше теоремы математического анализа в итоге может быть всегда смоделирована сложной функцией одного временного аргумента $t$. Уровень сложности итоговой зависимости очевидно определяется уровнем сложности взаимосвязей изучаемого явления, а также сложностью самих описывающих его факторов. Таким образом, любое экономическое явление динамично, т. е. развивается во времени, которое в конечном счете и является на сегодняшний день наиболее универсальным, агрегированным фактором экономической реальности, а само экономическое явление по сути это процесс. Концептуальное упрощение модели за счет рассмотрения одного независимого фактора сопряжено с математической сложностью описания изучаемого процесса, что вполне объясняется сложностью изучаемых реальных экономических явлений. Для эмпирических исследований любой процесс может рассматриваться как модель «черного ящика» и для обработки экспериментальных данных применяются либо классические логико-математические методы и алгоритмы, либо технологии компьютерного моделирования.

Поскольку все экономические факторы динамичны и их взаимосвязи также претерпевают изменения, то большинство удовлетворительных решений экономических проблем с помощью классических и неоклассических моделей состава и структуры в момент $t_{1}$ могут дать крайне неудовлетворительное решение в момент $t_{2}$, причем интервал $\Delta t=t_{2}-t_{1}$ имеет тенденцию к уменьшению, особенно если речь идет о периодах нестабильности, связанных с социально-экономическими трансформациями. То есть модели состава и структуры релевантны действительности на очень коротком временном отрезке. Время, потраченное на выявление значимых факторов (модель состава) и установление их функциональных взаимосвязей (модель структуры), а затем и на поиск решения поставленной задачи в рамках модели структуры, может оказаться несопоставимо большим по сравнению с $\Delta t$. Таким образом, описание современных экономических явлений доми- 
нирующими в классической экономической теории моделями состава и структуры, отражающими статические равновесные свойства экономической системы, дает удовлетворительный результат на краткосрочном горизонте или при достаточно идеализированных и маловероятных условиях стабильности структуры экономической системы. В современных исследованиях долгосрочного планирования и прогнозирования все чаще можно встретить выражения «структурные изменения», «структурные сдвиги», «структурная несбалансированность».

Для целей среднесрочного и долгосрочного анализа сложных экономических процессов использование традиционных методов эконометрики, статической оптимизации, производственных функций находится в противоречии с основными допущениями и условиями их применимости, когда время и протекающие процессы не имеют значения, а производится оценка текущих состояний с точки зрения равновесных цен и количеств. Вот как А. Маршалл (1993. С. 28) описывал связь понятия равновесия с понятием устойчивости, используя явную физическую аналогию: «...когда спрос и предложение пребывают в равновесии, количество товара, производимого в единицу времени, можно назвать равновесным количеством, а цену, по которой он продается, равновесной ценой. Такое равновесие является устойчивым, т. е. цена при некотором отклонении от него будет стремиться к возвращению в прежнее положение подобно тому, как маятник колеблется в ту и другую сторону от своей низшей точки». Несмотря на то, что понятие устойчивости ${ }^{7}$ давно формализовано на языке математики, экономисты неоклассической школы крайне редко используют его в своих практических расчетах, они лишь предполагают, иногда констатируют, что данным ключевым свойством система обладает. Однако для представителей эконофизики понятие устойчивости является предметом систематических исследований, одним из результатов которых стали модели мягкой и жесткой потери устойчивости для анализа состояния фондового рынка. Оценка времени устойчи-

7 Устойчивость определяется как способность системы сколь угодно долго оставаться около положения равновесия или на заданном многообразии. вости структуры экономической системы становится решающей при выборе соответствующего подхода к количественному анализу (классические статические факторные методы или же новые динамические процессносистемные подходы в моделировании).

Цифровая трансформация в результате комплексного использования цифровых ресурсов и технологий во всех сферах человеческой деятельности кардинально меняет социальные, политические, бизнесмодели и формирует новую цифровую среду. Современные цифровые технологии демонстрируют чрезвычайно высокий динамизм. Технология по сути есть реализация последовательных или параллельных процессов. Изменение технологии уничтожает одни процессы, регулирует и адаптирует другие, порождает новые. Используя современную терминологию, экономика представляет собой массивно-параллельную систему, образуемую одновременной деятельностью множества акторов, из целенаправленных действий которых формируется процесс (Артур, 2015). В экономике сегодня более актуальны вопросы формирования и изменения, а не размещения, ответы на которые логичнее искать в терминах процессов, а не количеств. Таким образом, процессно-системная методология, основанная на идеях динамизма эволюционных процессов, системном подходе к развитию современных экономических отношений в условиях цифровизации, трансформирует классическую иерархию «экономическая среда - система - агент», заменяя в ней структуру нижнего уровня «агент» на структуру «процесс», в триаду «экономическая среда - система - процесс».

Если раньше технология была эксклюзивным знанием, доступна ограниченному кругу субъектов экономики, то отличительной особенностью цифровых технологий является высокая степень их открытости. Они открыты широкому кругу потребителей, которые сами способны выступать в роли их производителей. Такое массовое одновременное потребление и производство цифровых технологий приводит к трансформации естественной среды. Сетевое взаимодействие систем разного уровня экономического пространства, с одной стороны, формируют эко- 
номическую среду, а с другой стороны, сами эти системы вынуждены встраиваться и находиться под влиянием среды, проявляя свойство ингерентности. Такое взаимодействие представляет собой рекурсивный цикл. Этот процесс требует от экономических систем непрерывной обеспеченности цифровыми ресурсами: техникой, услугами связи, носителями информации, технологическими решениями (ПО), что стимулирует рост и развитие радиоэлектронной промышленности, ИКТ сектора, сектора связи и обеспечивающих их НИОКР. Поэтому многие экономисты и политики называют «цифровую экономику» глобальным лоббистским проектом компаний микроэлектроники и ИКТ для поддержания высоких темпов роста, расширения рынков сбыта и увеличения их емкости, а также привлечения в отрасль масштабных инвестиций (Маевский, КирдинаЧэндлер, 2020). Таким образом, наблюдается феномен гибридной (естественно-искусственной) саморазвивающейся среды, все менее скованной проклятием ограниченности ресурсов ${ }^{8}$. «Проклятие ограниченности ресурсов» употребляется как метафора известному математическому выражению «проклятие размерности», которое возникло в результате несоответствия постоянно увеличивающейся размерности обрабатываемых данных вычислительным возможностям алгоритмов и/или мощностям вычислительных устройств $^{9}$. Сегодня поток генерируемых данных, подлежащих обработке, не измерим, поэтому классические математические алгоритмы уже не работают и им на смену приходят новые технологии больших данных (Big Data), снимающие в том числе и указанную математическую проблему.

Универсальность цифровых технологий позволяет применять их в разных системах с совершенно разными экономическими эффектами (одну и ту же платформенную технологию применяют в сфере торговли, финансов, образования, государственного управ-

8 Здесь речь идет о так называемых технологических и информационных ресурсах, которые по сути являются производной интеллектуальной деятельности и объективно не ограничены, но могут быть ограничены институциональными правилами.

9 В рамках теории сложности вычислений производится оценка вычислительной сложности через влияние объема входных данных на требование к временным ресурсам и объему памяти. ления) ввиду синтетических свойств целесообразности и эмерджентности системы. Платформы вслед за кластерами становятся объектом исследования мезоэкономистов, которые в своих исследовательских направлениях выделяют раздел «мезоэкономика сетевых структур» (Там же). В деятельности таких новых структур доминируют гибридные координационные механизмы (сетевые распределенные технологические решения в сочетании с институциональными принципами) материальных, технологических, финансовых и информационных потоков, поэтому платформы можно обозначить как институциональные технологические системы, которые также являются предметом пристального внимания представителей «институциональной мезоэкономики».

Следует отметить, что представители системного и эволюционного направлений экономической теории воспринимают и развивают идеи синергетики, которая трактует общий принцип социально-экономической динамики как «хаос на микроуровне - порядок на макроуровне». Синтез данного принципа и процессно-системного подхода объясняет формирование сетевой динамики из множества хаотичных процессов на микроуровне с учетом их свойств функциональности, стимулируемости, изменчивости и целенаправленного взаимодействия друг с другом для создания структуры макроуровня, устойчивой к внутренним и внешним дестабилизирующим воздействиям на протяжении определенного периода времени. Общая теория сложности объясняет образование структур разных уровней и то, как метасистема воздействует на обуславливающие ее объекты. Формирующее взаимодействие структур разных уровней представляет собой рекурсивный цикл, который можно представить в виде двухконтурных стрелок (Поддубная, 2020. С. 87). Таким образом, в рамках процессно-системного подхода взаимодействие процессов обеспечивает сетевую экономическую динамику (внутриуровневые горизонтальные связи), а системность - иерархическую организованность экономической среды (межуровневые вертикальные связи). Поэтому триаду «экономическая среда система - процесс» (Поддубная, 2020) с 
учетом описанных ее динамических особенностей предлагается называть иерархически-сетевой моделью EESP (аббревиатура «economical environment-system-process»). Данная модель позволяет интерпретировать экономическое развитие как эволюционную динамику, являющуюся результатом сетевых взаимодействий процессов разных уровней экономической системы. Поскольку в рамках динамического подхода любая социально-экономическая система инерционна и обладает исторической памятью, то элементы экономической системы постоянно обновляют свое состояние в соответствии с текущим моментом, учитывая и свое поведение в прошлом.

Рассмотрим общие принципы, некоторые особенности техники и инструментов моделирования, позволяющие реализовать концептуальные идеи нового подхода.

\section{Методы и технологии моделирования динамики социально-экономических систем}

Процессно-системный подход в иерархически-сетевой модели EESP трактует социально-экономическую систему как динамическую систему на любом уровне иерархии. Динамическая система - математическая абстракция или алгоритмически-цифровая формализация, предназначенная для описания и изучения эволюции систем во времени. Динамика некоторого явления описывается процессом перехода системы из одного состояния в другое. Состояние системы определяется множеством характеристик (координат) в некоторый фиксированный момент времени, т. е. в каждый момент времени система описывается вектором состояния $x=\left\{x_{1}, x_{2}, \ldots x_{n}, \ldots\right\}$ (конечным или бесконечным). Процесс перехода системы из одного состояния в другое (или изменения характеристик во времени) описывается оператором эволюции. Динамическая система считается описанной, если заданы начальное состояние и закон, по которому система переходит из начального состояния в другое.

Модель динамической системы в общем виде определяется совокупностью:

1) пространство состояний $X$, образующее полное метрическое пространство; пространство начальных состояний $X_{0} \subset X$;
2) множество моментов времени $T$; начальный горизонт $T_{0} \subset T$;

3 ) оператор эволюции $E_{t}$ (некоторое отображение $X \rightarrow X$ ), который каждому состоянию $x_{1} \in X$ в начальный момент времени $t_{0} \in T\left(x_{0}=x\left(t_{0}\right)\right)$ однозначно ставит в соответствие некоторое состояние $x(t) \in X$ в любой другой момент времени $t \in T$, т. е. $x(t)=E_{t} x\left(t_{0}\right)$.

Каждому состоянию системы соответствует изображающая точка. Совокупность изображающих точек определяет траекторию движения системы. Если множество $T=\left[t_{0}, t\right]$ (время изменяется непрерывно), то изучается континуальная (потоковая) динамическая система. Если множество $T=\left\{t_{0}, t_{1}, \ldots t_{n}, \ldots\right\}$ (время изменяется дискретно), то рассматривается динамическая система с дискретным временем. Несмотря на дискретность отдельных операций в экономических исследованиях, они чаще всего разновременные на достаточно большом промежутке времени, при их агрегировании часто переходят к потоковым модельным переменным и системам. Во второй половине XX в. Дж. Форрестером был предложен метод системной динамики, согласно которому динамика системы определяется структурой, описанной в виде взаимодействия потоков и контуров обратной связи (Forrester, 1961, 1969). Хорошо известна его компьютерная модель мировой динамики, ставшая основанием для принятия знаменитой экологической концепции развития человечества. «Мир-1» с точки зрения математической формализации являлась детерминированной нелинейной разностной моделью большой размерности, содержащей 5 глобальных переменных-уровней (вектор состояния), 7 переменных-темпов и ряд переменных-параметров (Forrester, 1971a).

Задача прогнозирования динамики изучаемой социально-экономической системы сводится к реализации глобальных этапов:

1) структурная идентификация оператора эволюции на основе имеющихся знаний и/или концептуальных гипотез об исследуемом объекте;

2) реконструкция параметров оператора эволюции по временным рядам ретроспективными или оперативными методами; 
3) определение по имеющейся информации об операторе эволюции и объекте в начальные моменты времени его будущего в любой последующий момент времени.

Первый этап является теоретическим, второй - эмпирическим, третий - оценочно-прогностическим. Выбор инструмента формализации оператора эволюции напрямую зависит от теоретической проработанности проблемы и качества ее «рыхлого» описания, т. е. актуальной концептуальной модели, в создании которой ключевую роль играет синтез современных идей и представлений о содержании экономической динамики. На этом этапе крайне важно не попасть в методологическую ловушку, когда делаются попытки настроить, откалибровать концептуальную модель с учетом разработанных, апробированных, устоявшихся инструментов и технологий модельных конструкций. С одной стороны, двигаясь в этом ложном направлении, исследователь часто вынужден задавать существенные ограничения и идеализированные условия в концептуальную конструкцию, которые не позволяют говорить об адекватности модели еще на теоретическом этапе прогнозирования. В этом случае имеет место бессодержательная математизация, оторванная от практики и реальных потребностей экономической политики. С другой стороны, понимая важность в современной экономической теории и практике количественного анализа, который дает эмпирическое объяснение научных гипотез и согласованности теоретических выводов с опытными данными, многие исследователи отказываются от развития новых концепций для описания социальных трансформаций, поскольку они не формализуются в рамках «жесткого» описания проблемы, фундамент которого построен на традиционном аппарате экономико-математического моделирования, разработанного для целей и задач описания преимущественно статических свойств социальноэкономических систем. В то же время все более значимым в объяснении и моделировании экономической динамики становится эволюционный взгляд на социально-экономи- ческие системы, направленный на объяснение причин их трансформаций и движущих сил развития. При этом формируется теоретический базис новых концептуальных моделей, которые, в свою очередь, инициируют необходимость разработки нового прикладного инструментария, включая методы количественного анализа, без которого прогностическая роль науки ничтожна.

Идея преодоления указанного методологического кризиса видится в двух направлениях: первое - использование нового математического аппарата для аналитической реализации моделей экономической динамики, второе - разработка новых алгоритмов и технологий обработки экспериментальных данных, лежащих в основе компьютерного моделирования.

Сформулируем основные положения для аналитического построения оператора эволюции при моделировании экономической динамики. Во-первых, в моделях любых социально-экономических трансформаций должны выделяться «быстрые» и «медленные» переменные, описывающие процессы, которые протекают в разных масштабах времени (скорости процессов различны). Во-вторых, экономическая инерционность и историческая память требуют наличия временных лагов в модельных переменных (чаще в «медленных»), поскольку состояние системы в будущий момент времени зависит не только от текущего состояния, но и от всей предыстории ее развития. Втретьих, экономическая динамика, особенно в переходных условиях, должна быть управляемой, т. е. должны быть формализованы механизмы целенаправленного достижения заданных или близких к ним состояний системы.

Таким образом, оператор эволюции $E_{t}$ социально-экономической системы в условиях трансформаций предлагается описывать в общем виде гибридной быстро-медленной системой с запаздываниями и управлением:

$$
\left\{\begin{array}{l}
\frac{d x(t)}{d t}=F_{1}(x(t), y(t), u(t)), \quad t>h_{l} \\
y(t)=F_{2}\left(x(t), y\left(t-h_{1}\right), y\left(t-h_{2}\right), \ldots, y\left(t-h_{l}\right), u(t)\right), t \geq h_{l},
\end{array}\right.
$$


где $x(t)$ - «быстрая» переменная, $y(t)-$ «медленная» переменная, $u(t)$ - экзогенное управление, постоянные $0<h_{1}<h_{2}<\ldots<h_{\text {i }}-$ временные лаги, $h_{i} \in R, \forall i \leq l, l \in N$.

Скорость «быстрой» переменной почти во всех точках пространства состояний $X$ много больше скорости «медленной» переменной, ввиду чего первое уравнение системы - дифференциальное, а второе - алгебраическое. В общем случае $x(t)$ и $y(t)$ многомерны, но динамические системы, содержащие более двух переменных, проявляют стохастический характер, что важно учитывать при моделировании. Для построения траектории движения системы необходимо задать начальный горизонт $T_{0}=\left[0, h_{i}\right)$ и пространство начальных состояний $X_{0}=\left\{x_{0}=x\left(h_{l}\right), y_{0}=\psi\left(t_{0}\right) \mid t_{0} \in T_{0}\right\}$, где $\psi(\cdot)$ принадлежит классу допустимых функций конкретной задачи. Наряду с таким общим принципом задания в модели временного лага $h_{l}$, как длительность временного интервала, на котором велось наблюдение за поведением системы (начальный горизонт $T_{0}$ ), трудоемкой и кропотливой задачей экспертов является уточнение данного параметра модели, на ее результат могут повлиять, например, качество собранных статистических данных, длительность ожидаемого прогноза и многие другие факторы, оценивать их влияние на корректировку параметра $h$, необходимо в условиях реальной моделируемой ситуации. В предложенной математической модели в описании структуры оператора эволюции присутствуют два «черных ящика» $F_{1}$ и $F_{2}$, их раскрытие осуществляется аналитическими или имитационными методами моделирования для конкретной прикладной задачи.

При идентификации оператора эволюции сложных социально-экономических систем для изучения практических задач исследователи сталкиваются с рядом проблем. Поскольку моделируемые системы уникальны и повторно невоспроизводимы во времени, то накопление и систематизация знаний о них затруднены, ввиду чего наблюдается слабая теоретическая изученность структуры и процессов системы. Coциально-экономические системы гораздо более сложны и тяжелы для понимания, нежели технические системы, они слабо предсказуемы и контринтуитивны ввиду большой доли субъективных экспертных знаний о них (Forrester, 1971b). При недостаточной изученности или слишком большой сложности экономического явления его аналитическое описание математической моделью часто либо невозможно, либо вызывает научный скепсис. В этом случае прибегают к экспериментальным методам компьютерного моделирования, в основе которых лежат алгоритмы и технологии обработки реальных данных.

Компьютерное моделирование - это технология анализа или синтеза сложных систем на основе использования их программных моделей, которые строятся поэтапно. На первом этапе происходит создание структурно-функциональной модели: реальную систему представляют в виде совокупности взаимодействующих элементов (блок-схем, графиков, диаграмм, таблиц, гипертекста и других структурированных, слабоструктурированных или неструктурированных данных). На втором этапе воспроизводятся (имитируются) различные режимы функционирования сложной системы. Описывают по определенным операционным правилам поведение отдельных элементов, которым ставят в соответствие некоторые программные компоненты, задающие переменные состояния, каждая комбинация которых передает конкретное состояние. Алгоритм изменения переменных состояний реализуется с помощью механизма продвижения модельного времени. Алгоритмы координации и обмена информацией моделируют механизм взаимодействия различных элементов между собой и с внешней средой.

Сегодня компьютерное моделирование мощнейшая технология эмпирического исследования. Ключевым моментом обработки экономических данных является его проведение в пассивном ретроспективном режиме с невозможностью многократного воспроизведения при одном и том же комплексе условий. Ввиду этого среди экономистов-аналитиков все чаще прослеживается тенденция отступления от кеплеровских стандартов эмпиризма, когда «тщательное наблюдение за движением» невозможно осуществить из-за его 
неповторимости, вследствие чего под временные ряды стараются подогнать статистические распределения и функциональные зависимости, собирая модель как конструктор из имеющегося набора деталей и соединяющих их элементов. Очевидно, что такая практика лишает исследователя возможности построения причинных моделей, объясняющих, а не обрабатывающих эмпирические данные.

Наиболее взвешенным и научно обоснованным, по нашему мнению, является смешанный аналитически-алгоритмический подход, когда причинная аналитическая модель, полученная априори в результате анализа экономических явлений и процессов, который изначально опирался на фундаментальные экономические теории (этап структурной идентификации), корректируется и калибруется в ходе имитационных экспериментов на реальных данных (этап реконструкции параметров).

$* \quad * \quad *$

В доминирующем на сегодняшний день классическом и неоклассическом направлении экономической теории акцент делается на статические свойства (целостность, открытость, внутренняя неоднородность, структурированность) экономических явлений с использованием в моделировании методологии факторного анализа. Общая теория равновесия является не просто математически элегантной: моделируя экономику, она воссоздает ее в нашем разуме, дает возможность сформировать ее образ, осмыслить экономику во всей ее целостности. Однако изящество равновесия зиждется на том, что в основе этих построений идеализированный, рационализированный мир, который искажает реальность, а их основополагающие предпосылки часто подбираются для аналитического удобства (Артур, 2015). Тотальный динамизм, многоаспектная значимость фактора времени, возрастающая сложность новых экономических структур и их взаимодействий, технологическая доминанта - черты цифровой трансформации, охватившей весь мир. Еще в середине ХХ в. Р. Солоу отмечал, что технология является не единственным агентом изменений в экономике, но, безусловно, основным. В ортодоксальной экономической теории на передний план выходят цены и количества, а технология, как основная движущая сила, отодвинута на задний план, упоминается лишь косвенно, в контексте более эффективного применения факторов. Статически равновесная парадигма экономического анализа для изучения экономических явлений в условиях развития должна дополняться новыми исследовательскими программами, в которых изучение механизмов взаимодействия процессов позволяет оценивать функциональность, стимулируемость, существование в изменяющейся среде, внутренний динамизм явления (динамические свойства). Уникальные свойства эмерджентности, ингерентности и целесообразности (синтетические свойства) каждой экономической системы определяют план ее развития и/или саморазвития. На основе синтеза идей институционально-эволюционного направления и экономики сложности формулируются новые концепции с использованием в моделировании процессно-системного подхода. В рамках данного подхода автором предложена иерархически-сетевая модель EESP, в которой акцент делается на исследовании динамических и синтетических свойств сложных адаптивных социально-экономических систем.

Статическая теория позволяет наблюдать изменения какого-либо экономического показателя за указанный период, но причины, механизмы и направления этих изменений остаются неизвестными, оценивать можно только количественную динамику экономических явлений. Динамический подход не только показывает количественные изменения, но и объясняет, какие экономические элементы и их соотношения вызывают такие изменения. В этом случае можно говорить о качественной динамике (Кондратьев, 1989). Поиску новых модельных конструкций и технологий, позволяющих формализовать качественные характеристики и их влияние на количественные показатели динамики экономических явлений, посвящены исследования ученых, разрабатывающих инструментальные кибернетические и синергетические подходы в рамках экономики сложности. 
В работе автор развивает идеи динамического подхода в моделировании для исследования социально-экономических явлений, который базируется на принципах детерминизма о закономерностях и причинной обусловленности всех явлений природы и общества. В рамках этого подхода предполагается, что если описать процессы, которые влияют на поведение рассматриваемой системы, и задать ее структуру и условия функционирования (т. е. определить оператор эволюции $E_{t}$, пространство начальных состояний $X_{0}$, начальный горизонт $T_{0}$ ), то можно однозначно предсказать все последующие состояния системы на горизонте прогнозирования $T$. Автором предложена математическая модель экономической динамики в условиях трансформации, в основе структурной идентификации оператора эволюции которой заложены три основные приниипа динамической сложности: наличие в модели «быстрых» и «медленных» переменных, временного лага, управляющего воздействия. Данная модель является математической абстракцией высокого уровня, конкретизация которой должна осуществляться в результате уточнения структуры оператора эволюции при моделировании конкретной задачи.

\section{СПИСОК ЛИТЕРАТУРЫ (REFERENCES)}

Артур У.Б. 2015. Теория сложности в экономической науке: иные основы экономического мышления. Terra Economicus. Т. 13. № 2. С. 1537. [Arthur W.B. 2015. Complexity economics: A different framework for economic thought. Terra Economicus. Vol. 13. No 2. PP. 15-37. (In Russ.)]

Глазьев С.Ю. 1993. Теория долгосрочного технико-экономического развития. Москва: ВлаДар. [Glazyev S.Yu. 1993. The theory of long-term technical and economic development. Moscow: VlaDar. (In Russ.)]

Жилин В.И. 2010. К вопросу самоорганизации в кибернетических и синергетических системах. Вестник Ленинградского государственного университета им. А.С. Пуикина. Т. 2. № 2. С. 142-
149. [Zhilin V.I. 2010. On the question of selforganization in cybernetics and synergistic systems. Vestnik Leningradskogo gosudarstvennogo universiteta im. A.S. Pushkina. Vol. 2. No 2. PP. 142-149. (In Russ.)]

Зеленков А.И. 2010. Синергетический стиль мышления и перспективы пластичной рациональности. Философия и социальные науки. № 1. C. 30-35. [Zelenkov A. 2010. Synergetic style of thinking and the prospects of flexible rationality. Filosofiya $i$ sotsial'nye nauki. No 1. PP. 30-35. (In Russ.)]

Кондратьев Н.Д. 1989. Проблемы экономической динамики. Москва: Экономика. [Kondratyev N.D. 1989. Problems of Economic Dynamics. Moscow: Economica. (In Russ.)]

Маевский В.И., Кирдина-Чэндлер С.Г. (Ред.). 2020. Мезоэкономика: элементы новой парадигмы. Москва: Институт экономики РАН. 392 c. [Maevsky V.I., Kirdina-Chandler S.G. (Eds.). 2020. Mesoeconomics: Elements of a New Paradigm. Moscow: Institut ekonomiki RAN. 392 p. (In Russ.)]

Маршалл А. 1993. Приниипь экономической науки. Т. II. Москва: Прогресс. [Marshall А. 1993. Principles of Economics. Vol. II. Moscow: Progress. (In Russ.)]

Поддубная О.Н. 2020. Теоретические и концептуальные подходы к моделированию экономической динамики в контексте цифровой трансформации. Белорусский экономический журнал. № 4. C. 79-90. [Poddubnaia O.N. 2020. Theoretical and conceptual approaches to economic dynamics modeling tailored to the digital transformation. Belorusskiy ekonomicheskiy zhurnal. No 4. PP. 7990. (In Russ.)]

Хакен Г. 2005. Информация и самоорганизачия. Макроскопический подход к сложным системам. Москва: КомКнига. [Haken G. 2005. Information and self-organization. Macroscopic approach to complex systems. Moscow: KomKniga. (In Russ.)]

Forrester J.W. 1961. Industrial dynamics. Waltham, MA: Pegasus Communications.

Forrester J.W. 1969. Urban dynamics. Waltham, MA: Pegasus Communications.

Forrester J.W. 1971a. World dynamics. Waltham, MA: Pegasus Communications.

Forrester J.W. 1971b. Counterintuitive Behavior of Social Systems. Technology Review. Vol. 73. No 3. PP. 52-68. 
In citation: Belorusskiy Ekonomicheskiy zhurnal. 2021. No 3. PP. 70-82.

Belarusian Economic Journal. 2021. No 3. PP. 70-82.

\title{
PROCESS-SYSTEM APPROACH IN STUDYING SOCIO- ECONOMIC SYSTEMS: FROM CONCEPTS TO MODELS
}

\section{Olesia Poddubnaia ${ }^{1}$}

\author{
Author affiliation: ${ }^{1}$ Belarusian State Economic University (Minsk, Belarus). \\ Corresponding author: Olesia Poddubnaia (poddubnaia.o@bseu.by).
}

ABSTRACT. By synthesizing current ideas of the institutional-evolutionary approach and the complexity economics, new approaches, expanding and developing the essential and meaningful understanding of the socio-economic system, are brought up for the discussion. Conceptual hierarchical-network model «economic environment-system-process» (EESP) is proposed. Within the given model a process-system approach for studying the dynamic and synthetic properties of complex socio-economic systems is described. General principles and technologies for identifying the evolution operator in modeling economic dynamics are described, by using the dynamic approach. Three basic principles of the dynamic complexity of economic systems in the context of social transformations are postulated. Mathematical model of economic dynamics in the form of a hybrid fast-slow system with delay and control is offered for consideration.

KEYWORDS: economical dynamics, institutional-evolutional concept, complexity economics, hierarchical-network model, evolution operator, fast-slow system.

JEL-code: B52, C53, O10.

DOI: $10.46782 / 1818-4510-2021-3-70-82$

Received 28.04.2021 